\title{
Emergency visits among end-of-life cancer patients in Taiwan: a nationwide population-based study
}

\author{
Yi-Hui Lee ${ }^{1,2+}$, Dachen Chu ${ }^{3,4}$, Nan-Ping Yang ${ }^{3,5+}$, Chien-Lung Chan ${ }^{6}$, Shun-Ping Cheng ${ }^{3,7}$, Jih-Tung Pai $i^{3,8+}$ \\ and Nien-Tzu Chang ${ }^{1 *}$
}

\begin{abstract}
Background: An increased number of emergency visits at the end of life may indicate poor-quality cancer care. The study aimed to investigate the prevalence and utilization of emergency visits and to explore the reasons for emergency department (ED) visits among cancer patients at the end of life.

Methods: A retrospective cohort study was performed by tracking one year of ambulatory medical service records before death. Data were collected from the cancer dataset of Taiwan's National Health Insurance Research Database (NHIRD).

Results: A total of 32,772 (19.2\%) patients with malignant cancer visited EDs, and 23,883 patients died during the study period. Of these, the prevalence of emergency visits in the mortality group was $81.5 \%$, and their ED utilization was significantly increased monthly to the end of life. The most frequent types of cancer were digestive and peritoneum cancers (34.8\%), followed by breast cancer (17.7\%) and head and neck cancers (13.3\%). Older patients, males, and those diagnosed with metastases, respiratory or digestive cancer were more likely to use ED services at the end of life. Use of an ED service in the nearest community hospital to replace medical centers for dying cancer patients would be more acceptable in emergency situations.
\end{abstract}

Conclusions: Our study provided population-based evidence related to ED utilization. An understanding of the reasons for such visits could be useful in preventing overuse of ED visits to improve the quality of end-of-life care.

Keywords: Cancer, Emergency visits, End-of-Life

\section{Background}

Cancer is the first leading cause of death in Taiwan and worldwide. 8.2 million people died from cancer in 2012, and the number of new annual cases will continue to increase by about $70 \%$ over the next 2 decades [1]. In the aging Taiwan society, there is also an increasing number of individuals dying of cancer who are covered by the national health insurance (NHI) system. Approximately 29,000 Taiwanese people died from cancer in 1997, and this increased to 40,300 in 2007, which accounted for 13 percent of deaths attributable to cancer $[2,3]$. There is a growing appreciation of cancer patients' needs for end-of-life (EOL) care services, especially for those with terminal illnesses. Cancer care tends to be aggressive in Taiwan, as shown by

\footnotetext{
* Correspondence: ntchang@ntu.edu.tw

†Equal contributors

'School of Nursing, College of Medicine, National Taiwan University, Taipei, Taiwan

Full list of author information is available at the end of the article
}

the increasing anticancer therapies, ICU stays, and hospital deaths $[4,5]$. Patients were found to be more likely to receive aggressive care if they were younger or lived in rural regions with fewer medical resources [6-8]. However, this might go against old Taiwanese beliefs. In Taiwan, "Dying in his/her own home is a good death" when it comes to the most important issue for terminal patients and their families in Taiwanese culture [9]. To respect the end of life medical wishes of patients, the Taiwan Hospice-Palliative Care Act was initiated in 2000. Its purpose was to help incurable patients to relieve their physical, psychological and spiritual suffering by palliative or supportive medical care, and it permits the legal termination of end-of-life patients' life-supporting devices based on safeguarding the rights and wills of these patients [10]. Whereas the NHI in Taiwan issues reimbursement to cancer patients who have the right

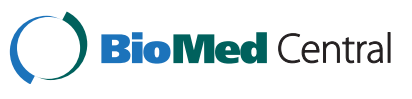

(c) 2015 Lee et al.; licensee BioMed Central. This is an Open Access article distributed under the terms of the Creative Commons Attribution License (http://creativecommons.org/licenses/by/4.0), which permits unrestricted use, distribution, and reproduction in any medium, provided the original work is properly credited. The Creative Commons Public Domain Dedication waiver (http://creativecommons.org/publicdomain/zero/1.0/) applies to the data made available in this article, unless otherwise stated. 
to refuse or receive aggressive medical treatment, the economic burden of cancer treatment in intensive care units presents an increasing trend [11].

For dying cancer patients, aggressive medical treatments and emergency department (ED) visits can result in distress and exhaustion. Abdominal pain, lung cancer, and dyspnea were reported to be the most common reasons for ED visits during the final six months of life and the final two weeks of life in Canada [12]. However, the available information is insufficient due to previous studies focusing on the experiences of these patients who visit an ED near the end of life being limited and having small sample sizes. Taiwan national claims data linked to the death registry database have been used in a similar field, but consideration of the aggressive treatment in EDs during the end of life and related factors were the focus of this study, which was to the best of our knowledge the first of its kind. The quality of EOL care received by terminal cancer patients and the affected factors have also seldom been explored in Asian countries [13]. Requesting an intensive ED service at the EOL stage will not improve the quality of EOL or prolong a patient's life, and also goes against the traditional beliefs regarding a good death in Taiwan. Therefore, the causes, symptoms and unavoidable events that lead to a terminal cancer patient visiting the emergency department deserve more attention. Reduction of futile aggressive medical treatment needs to be emphasized in order to improve the quality of EOL care [14,15]. To our knowledge, little is known about nationwide ED visits by terminal cancer patients when they are near the end of life [16]. Using Taiwan national claims data linked to the death registry database could be informative. In order to respond to current and emerging health issues apidly and effectively, the Taiwan National Health Insurance Bureau (NHIB) established a uniform system to control the quality of medical services and the coding database. The coverage of this single-payer program is greater than $99 \%$. This nationwide research database contains cancer registration and original claims data for reimbursement, enabling analysis by researchers. The study aimed to explore the prevalence and ED utilizations of all cancer patients in Taiwan in order to reveal the associations between cancer terminal patients and increased utilization of emergency visits near the end-of-life and to compare the distributions of diagnostic problems at EDs between cancer survivors and decedents.

\section{Methods}

\section{Data source, security, and quality control}

Taiwan launched a single-payer National Health Insurance (NHI) Program, financed jointly by payroll taxes, subsidies, and individual premiums, in 1995, and its coverage rate expanded to approximately $99 \%$ of the 23 million people in Taiwan who were enrolled in the NHI Research Database. All enrollees enjoy almost free access to healthcare, with a small co-payment by most clinics and hospitals $[17,18]$. The NHI Research Database includes nationwide population-based data with good quality control and representation and is provided to scientists in Taiwan for research purposes $[14,19]$. The information related to all subjects is encrypted using a double scrambling protocol for research purposes to protect the privacy of patients. Theoretically, it is impossible to query the data alone to identify individuals at any level using this database. All researchers who wish to use the NHIRD are required to sign a written agreement declaring that they have no intention of attempting to obtain information that could potentially violate the privacy of patients or care providers.

The study design was a retrospective cohort study. The protocol was evaluated by the NHRI (Application Number: 101020), who gave their agreement to the planned analysis of the NHIRD. The data protection and permission protocols were also approved by the Institutional Review Board (IRB) of Taoyuan General Hospital, which has been certificated by the Department of Health, Taiwan (IRB Approval Number: TYGH100021).

\section{Inclusion and exclusion criteria of the study population and definition}

In order to focus on ED utilization among end-of-life malignant cancer patients, the following criteria were identified: (1) emergency cases were excluded if they had used the services of emergency departments but their insurance numbers were not valid for tracing. (2) Patients with malignant cancer were diagnosed according to the International Classification of Diseases, Ninth Revision, Clinical Modification (ICD-9-CM), with first three-digit codes ranging from 140 to 208. The diagnosis codes of cancer types were grouped into six major categories. Their ICD-9-CM codes were defined as described in Table 1. (3) To find the death dates of subjects, another registered catastrophic illness dataset was linked to identify the survival status of the target population and perform the analyses [17]. (4) ED utilization of those with a diagnosis of cancer was identified from the NHIRD, confirmed by the case type code [20]. The definition of end-of-life is: "Any patient who has contracted a serious illness, diagnosed by a physician as at an incurable stage, and dying within 6 months, whose illness will inevitably lead to death in the near future". The study variable "urban and rural" was defined according to the degree of rurality, which was calculated by the Taiwan $\mathrm{NIH}$ research institute by employing parameters used in rurality analysis, which include population density (less than the mean per $\mathrm{km}^{2}$ ), the proportion\%) of elderly residents in the whole population, and proportion of actual workers in the area population [21]. The severity of each ED visiting was presented by triage scale. 
Table 1 Characteristics of cancer patients in Taiwan, $2008(n=170,271)$

\begin{tabular}{|c|c|c|c|c|c|}
\hline \multirow{2}{*}{$\overline{\text { Age, yr, }}$} & \multirow[t]{2}{*}{ All pat. $(n=170,271)$} & \multicolumn{2}{|c|}{ ED visitors $(n=32,772)$} & \multicolumn{2}{|c|}{ Mortality of ED visitors ( $n=23,883$ ) } \\
\hline & & & & & \\
\hline Mean (S.D.) & $61.0(14.9)$ & 64.0 & $(21.5)$ & 67.0 & $(14.4)$ \\
\hline \multicolumn{6}{|l|}{ Gender, No. (\%) } \\
\hline Male & $84215(49.5)$ & 20037 & 23.8 & 15551 & 18.5 \\
\hline Female & $86056(50.5)$ & 12735 & 14.8 & 8332 & 9.7 \\
\hline \multicolumn{6}{|l|}{ Types of cancer (ICD_9 codes) } \\
\hline Head and neck $(140-149,160,161)$ & 22600 & 4088 & 18.1 & 2819 & 12.5 \\
\hline Digestive and peritoneum (150-159) & 59223 & 15568 & 26.3 & 12245 & 20.7 \\
\hline Colorectal (153-154) & 33008 & 11371 & 34.5 & 9628 & 29.2 \\
\hline Other GI (150-152, 155-159) & 27483 & 4722 & 17.2 & 3032 & 11.0 \\
\hline Respiratory organs (162-165) & 16520 & 6523 & 39.5 & 5667 & 34.3 \\
\hline Lung cancer(162) & 13152 & 5820 & 44.3 & 5192 & 39.5 \\
\hline Other (163-165,190,192-194) & 2294 & 578 & 25.2 & 428 & 18.7 \\
\hline Bone, connective tissue, skin and breast (170-175) & 35697 & 3134 & 8.8 & 1674 & 4.7 \\
\hline Melanoma/sarcoma (170-172) & 3314 & 670 & 20.2 & 515 & 15.5 \\
\hline Breast (174) & 30118 & 2315 & 7.7 & 1052 & 3.5 \\
\hline Genitourinary organs (179-189) & 39475 & 5125 & 13.0 & 3050 & 7.7 \\
\hline Ovary (183) & 3737 & 519 & 13.9 & 335 & 9.0 \\
\hline Prostate (185) & 9782 & 1328 & 13.6 & 835 & 8.5 \\
\hline Metastases (196-199) & 10086 & 4837 & 48.0 & 4215 & 41.8 \\
\hline Lymphoma/leukemia (200-208) & 9403 & 2114 & 22.5 & 1334 & 14.2 \\
\hline
\end{tabular}

We used the cancer data file and death registrations from all insured beneficiaries to select the study subjects from Jan 12008 to Dec 31 2008. We investigated the ED utilizations of all cancer survivors and decedents. A total of 177,721 patients with a cancer diagnosis were identified, 35,477 cancer patients who died in 2008 and 142,244 survivors who were alive in 2008. 125,905 cancer survivors and 11,594 cancer decedents who died in 2008 without making an ED visit in the one-year observation period were excluded. For comparison without a lead time bias, 7,450 subjects in the survival group were also excluded as their survival status was missing or they did not survive or were not identified in the cancer dataset at Dec 31 2008. Thus, 170,271 patients with a cancer diagnosis were eligible and the ED visitors numbered 32,772. Among the ED visitors, the surviving cases totalled only 8,889. The other 23,883 cancer patients who died in 2008 were retrospective to investigate the whole one year records before they died. The flowchart of this study is shown in Figure 1.

\section{Statistical analysis}

Descriptive statistics are represented as numbers of cases, percentages, and means with standard deviation
(SD). Categorical data were analyzed using the Chisquare test. The Mantel-Haenszel Chi-square test was used to test for trends. The independent $\mathrm{t}$-test and 95\% confidence intervals ( $95 \% \mathrm{CIs}$ ) were used to analyze the significant differences in ED utilization between the two subgroups. All analyses of data were conducted using Microsoft SQL Server 2012 (Microsoft Corporation) and statistical calculations were performed using the Statistical Package for Social Sciences for Windows (SPSS 22.0).

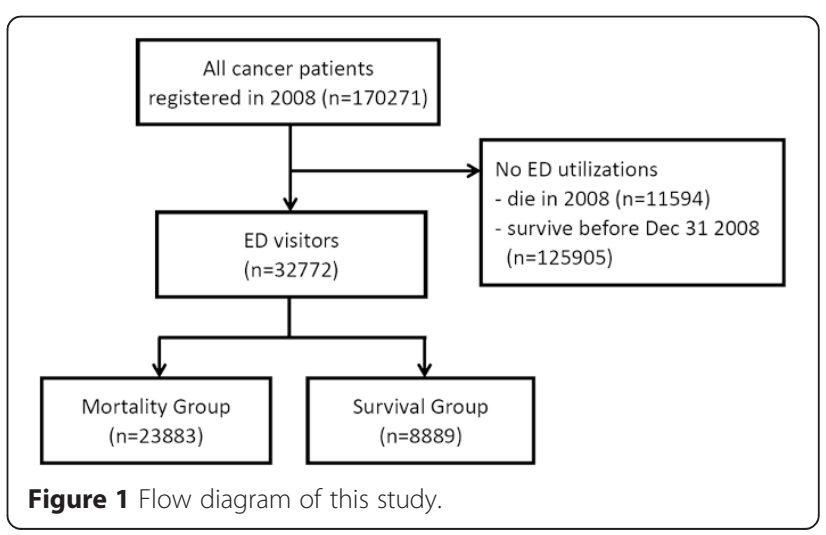




\section{Results}

\section{Characteristics of the cancer population}

The characteristics of the cancer patients and emergency cases that had used emergency department (ED) services before death are described in Table 1. A total of 170,271 patients with malignant cancer were identified, of whom 84,215 were male and 86,056 were female. Of those, the eligibility criteria identified 32,772 (19.2\%) patients who had utilized an ED and 23,883 (14.0\%) who died during the study period. The most frequent types of cancer in 2008 were digestive and peritoneum cancers $(34.8 \%)$, followed by breast cancer $(17.7 \%)$ and cancers of the head and neck (13.3\%). However, the most common medical problems leading to an ED visit were found to be metastases (ICD-9 codes 196-199), lung cancer (code 162), and digestive and peritoneum cancers (codes 150-159). The top three mortality rates of cancer patients who visited an ED within 6 months of the end of life were those with metastases $(41.8 \%)$, lung cancer (39.5\%) and colorectal cancer (29.2\%). Table 1 also shows the age and gender distributions of the enrolled subjects. Approximately $20 \%$ of the cancer patients made an ED visit in a year, the majority being male patients (23.8\%), older patients, and those near the end of life. The mean age of the mortality group was $67.0( \pm 14.4)$ years, and was significantly greater than that of the survival group $(p<0.001)$.

The characteristics of the cancer patients in 2008 are listed in Table 2, stratified by survival status. 35,477 (20.8\%) cancer patients who died during the study period were classified into the mortality group; the other 134,794 (79.2\%) survivors were alive in 2008 and were classified into the survival group. Among the mortality group, 23,883 dying patients used the majority of the ED resources, with 58,791 ED visits (81.5\%), and the mean number of visits was 2.46 per person (95\% confidence interval, CI: 2.43 to 2.49 ) in the last six months of life. The prevalence of ED utilization was $67.3 \%$ (95\% CI: $66.8 \%$ to $67.8 \%$ ) before death, which was significantly higher than that of the survival group (with a prevalence of $6.6 \%, 95 \%$ CI: $6.5 \%$ to $6.7 \%$ ). Among the survival group, the average number of ED visits was 1.51 per person (95\% CI: 1.47 to 1.55$)$ within the 6-month observational period, which was significantly lower than that of the mortality group $(p<0.001)$.

The patients in the mortality group who had used ED services had a more severe triage $(\mathrm{p}<0.001)$, and higher admission rate ( $4.9 \%$ vs $2.4 \%$ compared to the other group, $\mathrm{p}<0.001)$ that could lead to subsequent aggressive interventions and more treatment-related expenditure and greater length of stay in their last 6 months of life. Their total medical expenditure was significantly higher than the expenditure for the surviving cancer patients during the same observational period (US\$7,442.4 vs. US $\$ 4,243.6$,
Table 2 Utilization of emergency medical services among the cancer population in Taiwan

\begin{tabular}{|c|c|c|c|}
\hline & $\begin{array}{l}\text { Mortality group } \\
(n=35477)\end{array}$ & $\begin{array}{l}\text { Survival group } \\
(n=134794)\end{array}$ & $p$-value \\
\hline ED-visiting subjects & 23883 & 8889 & \\
\hline Age, mean (S.D.) & $67.0(14.4)$ & $59.5(14.7)$ & $<0.001$ \\
\hline \multicolumn{4}{|l|}{ Gender, n (\%) } \\
\hline Female & $8332(34.9)$ & $4403(49.5)$ & $<0.001$ \\
\hline Male & $15551(65.1)$ & $4486(50.5)$ & \\
\hline \multicolumn{4}{|l|}{$\begin{array}{l}\text { Types of hospital, } \\
\text { n (\%) }\end{array}$} \\
\hline $\begin{array}{l}\text { Community } \\
\text { hospital }\end{array}$ & 8980 (37.6) & 3138 (35.5) & $<0.001$ \\
\hline $\begin{array}{l}\text { Medical centers or } \\
\text { local hos. }\end{array}$ & $14903(62.4)$ & $5701(64.5)$ & \\
\hline \multicolumn{4}{|l|}{$\begin{array}{l}\text { ED hospitals with } \\
\text { hospice, } n(\%) \text { ward }\end{array}$} \\
\hline No & 9332 (39.1) & 3366 (38.1) & 0.052 \\
\hline Yes & $14551(60.9)$ & $5473(61.9)$ & \\
\hline \multicolumn{4}{|l|}{$\begin{array}{l}\text { Location of hospital, } \\
\mathrm{n}(\%)\end{array}$} \\
\hline Rural & $3624(15.2)$ & $1071(12.1)$ & $<0.001$ \\
\hline Urban & $20259(84.8)$ & 7768 (87.9) & \\
\hline $\begin{array}{l}\text { Prevalence of ED } \\
\text { visit }(95 \% \mathrm{Cl})\end{array}$ & $67.25(66.8-67.8)$ & $6.60(6.5-6.7)$ & $<0.001$ \\
\hline $\begin{array}{l}\text { Admission after the } \\
\text { ED visits }{ }^{a}, n(\%)\end{array}$ & $1160(4.9)$ & $210(2.4)$ & $<0.001$ \\
\hline \multicolumn{4}{|l|}{$\begin{array}{l}\text { Distributions of ED } \\
\text { visits triages }{ }^{\mathrm{b}}\end{array}$} \\
\hline Level I, count(\%) & $5647(11.3)$ & $575(4.3)$ & $<0.001$ \\
\hline Level II, count(\%) & $23521(47.0)$ & $5508(41.3)$ & \\
\hline Level III, count(\%) & $19444(38.9)$ & $6892(51.7)$ & \\
\hline Level IV, count(\%) & $1387(2.8)$ & $366(2.7)$ & \\
\hline
\end{tabular}

${ }^{a}$ Mean of those admission days were shown in text; Medical Cost, averaged number of ED visiting times, and prevalence of ED visit were shown in the text.

${ }^{b}$ Excluding missing data, the distributions of traditional ED triaged were presented because a new 5 -level triage scale had been proceed in Taiwan after 2010.

$p<0.001)$. In average, the length of stay 3.61 days $( \pm 3.47)$ was estimated for the mortality group, which was significantly more than the length of stay for the survival group (mean length of stay: $2.91 \pm 1.47$ ). Each kind of medical expenditure in emergency care, including diagnosis, treatment, drug costs and partial payment by themselves, each facet was observed in the mortality group approximately 1.4 to 2 -fold higher than in the survival group $(\mathrm{p}<0.001)$.

Dying patients could make ED visits for the relief of symptoms in the nearest community hospital rather than medical centers under emergency conditions. The odds ratio of the use of community hospitals for ED visits was significant at 1.10 (95\% CI: 1.04-1.15) in the mortality group 
patients. The rural location of the hospital (OR $=1.30,95 \%$ CI: 1.21-1.40) was also correlated with the ED utilization in emergency conditions, but hospitals with a hospice ward were not significantly related $(\mathrm{OR}=1.04,95 \% \mathrm{CI}: 0.99-1.10)$ among the cancer population.

\section{Comparisons of diagnostic categories of ED visits between cancer survivors and cancer decedents}

ED visits for cancer-related problems were identified from the cancer dataset of the National Health Insurance Research Database (NHIRD). Of 211,054 ED diagnostic problems during a 6-month period, there were 147,192 diagnoses in the mortality group and 63,862 diagnoses in the surviving group. The ICD-9-CM diagnoses codes were categorized as described in Table 3 . Besides the primary cancer diagnosis, the 15 most common diagnoses for ED visits were ranked by frequency and analyzed by the Chi-square test. The highly-ranked diagnoses differed slightly between the two groups. The first-ranked problem for visiting an ED in both groups was related to symptoms, signs, and ill-defined conditions (approximately 35\%). Visits related to the digestive system ranked second in both groups (18.5\% - 19.0\%). However, the third-ranked problem among the cancer survivors and decedents differed. The respiratory system ranked third (12.3\%) among the mortality group during the last six months of life, while genitourinary problems (11.2\%) ranked third in the cancer survivors. Besides symptoms, signs, and the primary cancer, the following chief problems in the mortality group were observed significantly less often than in the surviving group: genitourinary system, circulatory system, nervous system, mental disorders, musculoskeletal system and congenital abnormalities.

Of all ED utilizations among all cancer patients, the second top ranked problem was diseases of the digestive system, but there was no statistically significant difference in ED utilization between the mortality group and the survival group (19.0\% v.s. $18.5 \%, p=0.147)$. Among the mortality group, patients with digestive problems had a high number of ED visits during their last six months of life in Taiwan.

Analyzing our cancer data, the prevalence of ED utilization for cancer patients in Taiwan is illustrated in Figure 2. The distributions of ED utilization in various months in the two compared groups (mortality vs. survival groups) were obviously different in every threemonth period in 2008. Among the mortality group, a higher prevalence of ED visits was found in the last 12month period. Furthermore, when the time was closer to the end of life, ED visits became more frequent in the cancer decedents. A Mantel-Haenszel extension of the chi-square test for trend was significant $(p<0.001)$.

\section{Discussion}

Palliative care is an essential and fundamental component of cancer care [22]. In end-of-life care, emergency staffs (including physicians and nurses) are asked to receive hospice patients. ED staff need to recognize the complexities involved for the best management of a

Table 3 Distributions of ED utilization stratified by diagnostic category and survival status $(\mathbf{n}=32,772)$

\begin{tabular}{|c|c|c|c|c|}
\hline \multicolumn{2}{|c|}{ Summed disease categories of all ED utilizations } & \multirow{2}{*}{$\begin{array}{l}\text { Mortality group } \\
(n=23,883) \\
(\%)\end{array}$} & \multirow{2}{*}{$\begin{array}{l}\text { Survival group } \\
(n=8889) \\
(\%)\end{array}$} & \multirow[t]{2}{*}{$p$ value } \\
\hline \multicolumn{2}{|c|}{ ICD_9 codes diagnostic category } & & & \\
\hline 001-139 & Infectious and parasitic diseases & 4.35 & 3.35 & $<0.001$ \\
\hline $140-239$ & Neoplasms & 100 & 100 & 1.000 \\
\hline 240-279 & Endocrine, nutritional and metabolic diseases and immunity disorders & 8.20 & 6.45 & $<0.001$ \\
\hline 280-289 & Diseases of the blood and blood-forming organs & 4.44 & 3.07 & $<0.001$ \\
\hline 290-319 & Mental disorders & 0.76 & 1.63 & $<0.001$ \\
\hline 320-389 & Diseases of the nervous system and sense organs & 1.48 & 2.38 & $<0.001$ \\
\hline $390-459$ & Diseases of the circulatory system & 6.55 & 7.86 & $<0.001$ \\
\hline $460-519$ & Diseases of the respiratory system & 12.31 & 8.40 & $<0.001$ \\
\hline $520-579$ & Diseases of the digestive system & 19.01 & 18.54 & 0.147 \\
\hline $580-629$ & Diseases of the genitourinary system & 7.44 & 11.17 & $<0.001$ \\
\hline 680-709 & Diseases of the skin and subcutaneous tissue & 1.32 & 2.66 & $<0.001$ \\
\hline 710-739 & Diseases of the musculoskeletal system and connective tissue & 2.21 & 2.64 & $<0.001$ \\
\hline 740-759 & Congenital abnormalities & 0.08 & 0.13 & 0.018 \\
\hline $760-779$ & Certain conditions originating in the perinatal period & 0.02 & 0.02 & 0.606 \\
\hline 780-799 & Symptoms, signs, and ill-defined conditions & 34.52 & 39.25 & $<0.001$ \\
\hline 800-999 & Injury and poisoning & 2.34 & 4.17 & $<0.001$ \\
\hline
\end{tabular}



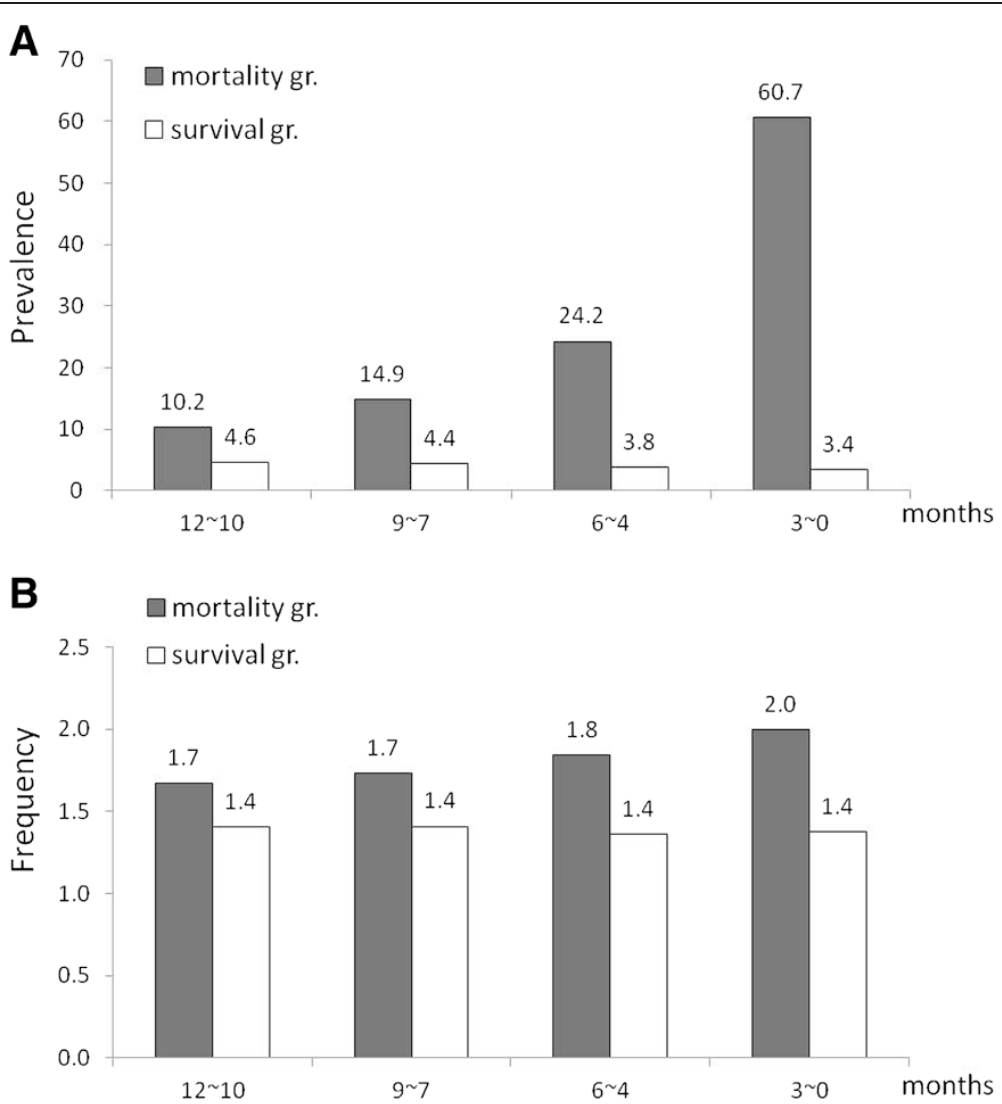

Figure 2 ED utilizations in the mortality and survival groups. A. Prevalence. B. Frequency of ED visiting times.

growing population of hospice patients. This study addressed the suffering of EOL patients using a nationwide dataset, and also described the visits of surviving patients with terminal cancer who presented to EDs [23]. Although palliative care is generally offered with the standard services in various settings, systematic characteristics influence the ability to implement the delivery of care for terminally ill patients [24]. Based on this evidence, an integrated community care service model should be developed, which integrates palliative care throughout the cancer trajectory [22].

Health system characteristics and patient cultural factors may play roles in the observed differences [6]. Several studies in Western countries have emphasized that hospice care at home is best for dying patients with cancer rather than aggressive treatment. However, Taiwan health insurance covers almost all medical services provided in hospitals for cancer patients, and therefore there are fewer economic barriers in end-of-life care. The barriers to ED utilization are decreased for cancer patients owing to healthcare fee remission. A study evaluating the medical utilization of the Taiwan NHI system revealed that on average, a person had 13.4 physician consultations and visited 3.9 healthcare facilities in a year, and $17.3 \%$ of the studied cohort had visited different healthcare facilities on the same day [25]. A copayment method was introduced into Taiwan's healthcare system, which could add some economic load and decrease inappropriate use and overuse of medical resources. A study analyzing the correlation between NHI-defined catastrophic illness and remitting medical expenditure revealed that patients with any certificated catastrophic illness tended to have significantly more ED visits and higher ED costs due to untypical medical complaints [16]. Furthermore, cancer care could potentially tend to become aggressive, as shown by the frequent ED visits and admissions to intensive care units at patients' end of life [5,13]. Advanced care planning emphasizes value-based care and enables further development towards hospice and palliative care [24].

The primary purpose of this study was to explore the prevalence of ED utilization and the expenditure on emergency care among the Taiwanese cancer population. A greater emergency medical expenditure at the end of a six-month period was observed in the mortality group, which included treatment-associated expenditure and drug-associated expenditure in Taiwan's EDs. The average costs were significantly higher in the mortality group than in the survival group (US\$7,442.4 vs. 4,243.6; $p<0.001$ ). The high utilization and medical costs were similar to those 
reported in the US and Canada [26]. One study focused on patients who died of cancer in 2001; $27.6 \%$ had at least one ER visit and 5.4\% had an ICU visit in the last two weeks of life, and Barbera [15] indicated that the high proportion of intensive therapies for cancer patients very near to death represent poor-quality end-of-life care. Another similar result was also found in North Carolina in 2008: there were 194,017 ED visits by 76,759 patients with cancer, and among these visits, those paying the most were with Medicare (for 52.4\%) and Medicaid (for 12.1\%). Hence, cancer treatment costs are increasing at an unprecedented rate $[16,26]$. The economics of health care need to be emphasized, and home-based palliative care needs to be considered in order to decrease the emergency expenditure and number of visits at the end of life [27,28]. Palliative care is one component of rural practice that requires interprofessional collaboration. Members of the care team need to have a sense of accountability and mutual respect. Thereby the autonomic team of rural practitioner devote their time to palliative care. That would be one of potential reasons that access to care and service utilization might be employed [29].

Age and gender effects were observed in end-of-life ED utilization. In the present nationwide population study, and some studies in the US and Canada, patients were found to be more likely to receive aggressive cancer care at the end of life if they were male, younger, lived in rural regions, had a higher level of comorbidity, or had breast, lung, or hematologic malignancies $[6,30]$. However, another study debated whether cancer-related ED visits are more likely to be made by male patients and those of an older age [31].

The results of Leak's study also indicated that the chief complaints among cancer ED visitors were in those dying of respiratory and gastrointestinal symptoms and those with a mental status change according to the 37,760 cancer-related ED visits contained in the North Carolina Disease Event Tracking and Epidemiologic Collection Tool [31]. The results were similar to the reason distributions of the identified categories of patients with cancer presented in the current study. Cardiopulmonary arrest, respiratory distress, and shortness of breath are well-known symptoms among end-of-life cancer patients [32]; however, we need to be aware that abdominal pain and gastrointestinal issues are also some of the most frequent problems for cancer ED visits during the final six months of life [16]. The results of Burge's study in Canada indicated that patients with a low continuity of care made 3.9 more ED visits than those experiencing a high continuity of care. The significant association between continuity of care and ED visits at the end of life among cancer patients needs to be emphasized [28]. ED visits can be prevented with appropriate home support and education and a focus on symptom management
[9,33], and keeping patients more comfortable at home under palliative care indicates good-quality end-of-life cancer care [12,34].

The strength of the present study was the nationwide epidemiological survey to analyze the end-of-life care for the Taiwanese cancer population. Some limitations exist: if a patient suffered prolonged hospitalization in his or her last 6 months of life, we may underestimate the ED utilization. An additional limitation of claims data mining is that there is a loss of detail. Possible confounders were not controlled in the present study, including socio-economic influences and family support, which were unable to be extracted from the epidemiological data. If patients do not have valid insurance, they may die in distress. Further investigation using an integrated community care model throughout the cancer trajectory survey is recommended.

\section{Conclusion}

Palliative care is an important issue for Taiwan cancer patients and deserves more attention. Population-based data can further provide evidence to support the development of a cancer policy and EOL care services. Our study provided population-based evidence of ED visits by patients with cancer in Taiwan. The results also provided empirical evidence regarding what EOL cancer patients perceive as being their needs. Patients with malignant cancer diagnoses had significantly more ED visits and higher ED medical costs. An understanding of the problems leading to such visits could be useful in preventing the overuse of ED visits to improve the quality of EOL care.

\section{Competing interests}

The authors declare that they have no competing interests.

\section{Authors' contributions}

Conceived and designed the experiments: YHL, JTP, NTC. Analyzed the data: NPY, CLC. Wrote the paper: YHL, DCC, NPY, SPC, JTP, NTC. In general, Y-HL, J-TP, and N-PY contributed equally to this work. All authors read and approved the final manuscript.

\section{Acknowledgement}

This study was based on data from the National Health Insurance Research Database and funded by the Ministry of Health \& Welfare of Taiwan (No. TYGH10101).

\section{Author details}

${ }^{1}$ School of Nursing, College of Medicine, National Taiwan University, Taipei, Taiwan. ${ }^{2}$ Department of Nursing, School of Nursing, College of Medicine, Chang-Gang University, Taoyuan, Taiwan. ${ }^{3}$ Institute of Public Health, College of Medicine, National Yang-Ming University, Taipei, Taiwan. ${ }^{4}$ Department of Neurosurgery, Taipei City Hospital, Taipei, Taiwan. ${ }^{5}$ Department of Medical Research, Keelung General Hospital, Ministry of Health \& Welfare, Keelung, Taiwan. ${ }^{6}$ Department of Information Management, Yuan-Ze University, Taoyuan, Taiwan. ${ }^{7}$ Department of Rehabilitation, Taoyuan General Hospital, Ministry of Health \& Welfare, Taoyuan, Taiwan. ${ }^{8}$ Department of Oncology \& Internal Medicine, Taoyuan General Hospital, Ministry of Health \& Welfare, Taoyuan, Taiwan. 
Received: 30 October 2014 Accepted: 31 March 2015

Published online: 09 May 2015

\section{Reference}

1. WHO. Cancer. In.2015 [http://www.who.int/mediacentre/factsheets/fs297/ en/index.html]

2. Chen LF, Ho HC, Su YC, Lee MS, Hung SK, Chou P, et al. Association between provider volume and healthcare expenditures of patients with oral cancer in Taiwan: a population-based study. PLoS One. 2013;8(6):e65077.

3. Chuang RB, Lee IF, Chiu TY, Wang JZ, Lai YL, Hsiao SC, et al. A preliminary experience of hospice shared-care model in Taiwan [in Chinese]. Taiwan J Hosp Palliat Care. 2005;10(Taiwan J Hosp Palliat Care):234-42.

4. Seow H, Barbera L, Howell D, Dy SM. Using more end-of-life homecare services is associated with using fewer acute care services: a population-based cohort study. Med Care. 2010;48(2):118-24.

5. Tang ST, Wu SC, Hung YN, Huang EW, Chen JS, Liu TW. Trends in quality of end-of-life care for Taiwanese cancer patients who died in 2000-2006. Ann Oncol. 2009;20(2):343-8.

6. Ho TH, Barbera L, Saskin R, Lu H, Neville BA, Earle CC. Trends in the Aggressiveness of End-of-Life Cancer Care in the Universal Health Care System of Ontario, Canada. J Clin Oncol. 2011;29(12):1587-91.

7. Rosenwax LK, McNamara BA, Murray K, McCabe RJ, Aoun SM, Currow DC. Hospital and emergency department use in the last year of life: a baseline for future modifications to end-of-life care. Med J Aust. 2011;194(11):570-3.

8. Tang ST, Chen ML, Huang EW, Koong SL, Lin GL, Hsiao SC. Hospice utilization in Taiwan by cancer patients who died between 2000 and 2004. J Pain Symptom Manage. 2007;33(4):446-53.

9. Cheng SY, Dy S, Hu WY, Chen CY, Chiu TY. Factors affecting the improvement of quality of dying of terminally ill patients with cancer through palliative care: a ten-year experience. J Palliat Med. 2012;15(8):854-62.

10. Yang NP, Jen I, Chuang SY, Chen SH, Chou P. Screening for low bone mass with quantitative ultrasonography in a community without dual-energy X-ray absorptiometry: population-based survey. BMC Musculoskelet Disord. 2006;7:24.

11. Lee JM, Turini M, Botteman MF, Stephens JM, Pashos CL. Economic burden of head and neck cancer. A literature review. Eur J Health Econ. 2004;5(1):70-80.

12. Barbera L, Taylor C, Dudgeon D. Why do patients with cancer visit the emergency department near the end of life? Can Med Assoc J. 2010;182(6):563-8.

13. Gilbertson-White $S$, Aouizerat BE, Jahan T, Miaskowski C. A review of the literature on multiple symptoms, their predictors, and associated outcomes in patients with advanced cancer. Palliative Supportive Care. 2011;9(1):81-102.

14. Earle CC, Park ER, Lai B, Weeks JC, Ayanian JZ, Block S. Identifying potential indicators of the quality of end-of-life cancer care from administrative data. J Clin Oncol. 2003;21(6):1133-8.

15. Barbera $L$, Paszat $L$, Chartier $C$. Indicators of poor quality end-of-life cancer care in Ontario. J Palliat Care. 2006;22(1):12-7.

16. Mayer DK, Travers D, Wyss A, Leak A, Waller A. Why do patients with cancer visit emergency Departments? Results of a 2008 Population Study in North Carolina. J Clin Oncol. 2011;29(19):2683-8.

17. Yang NP, Lee YH, Chung CY, Hsu JC, Yu IL, Chang NT, et al. Comparisons of medical utilizations and categorical diagnoses of emergency visits between the elderly with catastrophic illness certificates or not. BMC Health Serv Res. 2013. In press.

18. Taiwan NHI Information for the public: essential data of ensured affair. [Available at: http://w3.nhri.org.tw/nhird//en/index.htm]

19. Chan $\mathrm{CL}$, Lin W, Yang NP, Huang HT. The association between the availability of ambulatory care and non-emergency treatment in emergency medicine departments: a comprehensive and nationwide validation. Health Policy. 2013;110(2-3):271-9.

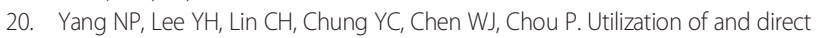
expenditure for emergency medical care in Taiwan: a population-based descriptive study. J Epidemiol. 2009;19(1):41-8.

21. Pozet A, Westeel V, Berion P, Danzon A, Debieuvre D, Breton JL, et al. Rurality and survival differences in lung cancer: a large population-based multivariate analysis. Lung Cancer. 2008;59(3):291-300.
22. Partridge AH, Seah DS, King $T$, Leighl NB, Hauke $R$, Wollins DS, et al. Developing a service model that integrates palliative care throughout cancer care: the time is now. J Clin Oncol. 2014;32(29):3330-6.

23. Zieske M, Abbott J. Ethics seminar: the hospice patient in the ED: an ethical approach to understanding barriers and improving care. Acad Emerg Med. 2011;18(11):1201-7.

24. Klinger CA, Howell D, Zakus D, Deber RB. Barriers and facilitators to care for the terminally ill: a cross-country case comparison study of Canada, England, Germany, and the United States. Palliat Med. 2014;28(2):111-20.

25. Chen TJ, Chou LF, Hwang SJ. Patterns of ambulatory care utilization in Taiwan. BMC Health Serv Res. 2006;6:54.

26. Kolodziej M, Hoverman JR, Garey JS, Espirito J, Sheth S, Ginsburg A, et al. Benchmarks for value in cancer care: an analysis of a large commercial population. J Oncol Pract. 2011;7(5):301-6.

27. Klinger CA, Howell D, Marshall D, Zakus D, Brazil K, Deber RB. Resource utilization and cost analyses of home-based palliative care service provision: The Niagara West End-of-Life Shared-Care Project. Palliat Med. 2013;27(2):115-22.

28. Burge F, Lawson B, Johnston G. Family physician continuity of care and emergency department use in end-of-life cancer care. Med Care. 2003;41(8):992-1001.

29. Gaudet A, Kelley ML, Williams AM. Understanding the distinct experience of rural interprofessional collaboration in developing palliative care programs. Rural Remote Health. 2014;14(2):2711.

30. Warren JL, Barbera L, Bremner KE, Yabroff KR, Hoch JS, Barrett MJ, et al. End-of-Life Care for Lung Cancer Patients in the United States and Ontario. J Natl Cancer Inst. 2011;103(11):853-62.

31. Leak A, Mayer DK, Wyss A, Travers D, Waller A. Why do cancer patients die in the emergency Department? an analysis of 283 Deaths in NC EDs. Am J Hosp Palliat Med. 2013;30(2):178-82.

32. Seow H, Barbera L, Sutradhar R, Howell D, Dudgeon D, Atzema C, et al. Trajectory of performance status and symptom scores for patients with cancer during the last six months of life. J Clin Oncol. 2011;29(9):1151-8.

33. Brink $P$, Partanen L. Emergency Department use among end-of-life home care clients. J Palliat Care. 2011;27(3):224-8.

34. Follwell M, Burman D, Le LW, Wakimoto K, Seccareccia D, Bryson J, et al. Phase II study of an outpatient palliative care intervention in patients with metastatic cancer. J Clin Oncol. 2009;27(2):206-13. 